\title{
Substitution Mapping of the Major Quantitative Trait Loci Controlling Stigma Exsertion Rate from Oryza glumaepatula
}

Quanya Tan, Tuo Zou, Mingmin Zheng, Yuerong Ni, Xin Luan, Xiaohui Li, Weifeng Yang, Zifeng Yang, Haitao Zhu, Ruizhen Zeng, Guifu Liu, Shaokui Wang, Xuelin Fu* and Guiquan Zhang ${ }^{*}$ (D)

\begin{abstract}
Background: Stigma exsertion rate (SER) is a key determinant for the outcrossing ability of male sterility lines (MSLs) in hybrid rice seed production. In the process of domestication, the outcrossing ability of cultivated rice varieties decreased, while that of wild Oryza species kept strong. Here, we detected the quantitative trait loci (QTLs) controlling SER using a set of single-segment substitution lines (SSSLs) derived from O. glumaepatula, a wild Oryza species.

Results: Seven QTLS for SER were located on 5 chromosomes. aSER-1a and aSER-1b were located on chromosome 1. qSER-3a and qSER-3b were mapped on chromosome 3, and aSER-3b was further located at an estimated interval of $898.8 \mathrm{~kb}$ by secondary substitution mapping. qSER-5, qSER-9 and qSER-10 were identified on chromosomes 5, 9 and 10, respectively, and QSER-9 was delimited to an estimated region of $551.9 \mathrm{~kb}$ by secondary substitution mapping. The additive effects of the 7 QTLs ranged from $10.6 \%$ to $14.8 \%$, which were higher than those of most loci for SER reported previously.
\end{abstract}

Conclusions: qSER-1 $a$ and qSER-1b are novel loci for SER on chromosome 1. All of the 7 QTLs have major effects on SER. The major QTLS of SER will help to develop MSLs with strong outcrossing ability.

Keywords: Stigma exsertion, Outcrossing, Quantitative trait locus, Single-segment substitution line, Wild species, Rice

\section{Background}

Rice is a staple food supplying more than $20 \%$ calories for the world population, thus playing a key role in sustaining world food security (Fitzgerald et al. 2009). The success of hybrid rice commercialization has greatly improved the yield of rice (Qian et al. 2016). However, cultivated rice is predominantly self-fertilizing with less than $1 \%$ natural cross-pollination (Virmani and Athwal 1973). The outcrossing ability of cultivated rice varieties diminished along with changes in the morphology of rice flowers during the process of domestication (Parmar et al. 1979). Previous studies have shown that the exserted stigma, as a

\footnotetext{
*Correspondence: fuxuelin@scau.edu.cn; gqzhang@scau.edu.cn Guangdong Provincial Key Laboratory of Plant Molecular Breeding, State Key Laboratory for Conservation and Utilization of Subtropical Agro-Bioresources, South China Agricultural University, Guangzhou 510642, China
}

main mating organ, can survive for several days after flowering (Kato and Namai 1987), and then get more opportunities to catch pollens (Xu and Shen 1988; Marathi and Jena 2015). Therefore, the stigma exsertion rate (SER) is a key determinant for the outcrossing ability of male sterility lines (MSLs) in hybrid rice seed production.

Wild Oryza species has strong ability of outcrossing. It is reported that perennial wild rice has higher outcrossing than annual types (Oka and Morishima 1967). Cultivated rice tends to have a shorter stigma than the annual wild species. The annual wild species has shorter stigma than their perennial progenitors (Virmani and Athwal 1973; Parmar et al. 1979; Marathi et al. 2015). The wide variability for stigma, style, and their total lengths in wild species of rice might have developed simultaneously during evolution and domestication (Marathi et al. 2015). In the past two 
decades, many of QTLs controlling SER and related traits have been located on 12 chromosomes of rice genome from various genetic resources (Marathi and Jena 2015; Zhou et al. 2017a; Liu et al. 2019). However, only 2 wild Oryza species were used to identify QTLs of SER until now, and O. rufipogon was most commonly used. Xiong et al. (1999) reported a QTL for extruding stigma on chromosome 6 using a $\mathrm{F}_{2}$ population derived from a cross between $O$. sativa, Aijiao Nante, and O. rufipogon, P16. Li et al. (2001) found two QTLs of SER on chromosomes 5 and 8 by a backcross- $\mathrm{F}_{1}$ population between O. rufipogon and Guichao 2 , an elite indica rice. Uga et al. (2003a) identified two QTLs for the rate of exserted stigma between the indica line Pei-kuh and W1944 of O. rufipogon. Huang et al. (2012) found 8 QTLs for SER using the $271 \mathrm{~F}_{2}$ lines from the cross between $O$. sativa, Guangluai-4, and O. rufipogon. Bakti and Tanaka (2019) detected five QTLs for SER using a $\mathrm{F}_{2}$ population generated from a cross between the japonica rice cultivar 'Akidawara' and 'W0120' of O. rufipogon. From another wild species, O. longistaminata, several QTLs of SER were identified from an introgression line of the wild species in the genetic background of Asian cultivated rice (Li et al. 2010).

O. glumaepatula is one of the AA-genome wild Oryza species indigenous to Central and South America (Doi et al. 2008). Recently, Stein et al. (2018) elucidated that $O$. glumaepatula is the sister group only of African $O$. barthii and $O$. glaberrima based on the genome sequence alignment. Several hybrid pollen sterility genes between $O$. sativa and O. glumaepatula have been identified, such as S12, S22, S23, S27 and S28 (Sano 1994; Sobrizal et al. 2000a, b; Sobrizal and Yoshimura 2001, 2002; Sakata et al. 2014; Fang et al. 2019). It was found that the endosperm of O. glumaepatula had the high levels of total protein, albumin, and glutelin protein fractions and amino acids, showing the potential to increase the nutritional quality of rice storage protein (Santos et al. 2013). O. glumaepatula also showed the rapid internodal elongation potential under partial submergence (Sasayama et al. 2018), as well as its important yield and yield component traits (Zhang et al. 2015; Bhatia et al. 2017). It is important to note that $O$. glumaepatula has a longer stigma length, which is favorable for outcrossing (Marathi et al. 2015).

In order to detect QTLs for traits of agronomic importance in O. glumaepatula, 168 SSSLs were developed by using $O$. glumaepatula as donors and Huajingxian 74 (HJX74), an indica variety of $O$. sativa as recipient. The total length of the substituted segments in the set of SSSLs was $3636.4 \mathrm{cM}$, covering 12 chromosomes (Zhao et al. 2019). In the present study, we used the set of SSSLs to identify QTLs for SER. Seven of the QTLs were located on 5 chromosomes. The QTLs had higher additive effects. These major QTLs of SER from $O$. glumaepatula will be helpful for developing of MSLs with strong outcrossing ability.

\section{Results}

SER in the SSSLs Derived from O. glumaepatula

Compared with the HJX74 recipient, O. glumaepatula, the donor of SSSLs, showed higher SER. On the average of 5 cropping seasons, SER in the donor was $68.6 \%$, while $29.6 \%$ in the recipient (Fig. 1a-c and Additional file 1: Table S1). Firstly, a set of 168 SSSLs derived from $O$. glumaepatula was investigated for SER and 9 of the SSSLs with higher SER were selected. The 9 SSSLs were then tested their SER for 5 cropping seasons. Compared with the HJX74 recipient, the 9 SSSLs showed higher SER at the $P \leq 0.001$ level in every cropping season. Average SER of the 9 SSSLs in 5 cropping seasons were from $48.1 \%$ to $60.8 \%$ with $18.5-31.2 \%$ higher than that of the control HJX74 (Fig. 1c and Additional file 1: Table S1). Analysis of variance (ANOVA) of the 9 SSSLs in 5 cropping seasons showed that the $F$-values of lines (9 SSSLs), seasons (5 cropping seasons) and lines by seasons showed significant difference at the $P \leq 0.001$ level (Additional file 1: Table S2). However, The SER between the first cropping season (FCS) from late February to middle July and the second cropping season (SCS) from late July to middle November showed no significant difference (Fig. 1d).

The chromosomal substituted segments from O. glumaepatula in the 9 SSSLs were detected by molecular markers. The substituted segments in the 9 SSSLs were detected on chromosomes 1, 3, 5, 9 and 10, respectively. The estimated lengths of substituted segments were from $1467.0 \mathrm{~kb}$ to $16,410.5 \mathrm{~kb}$ (Additional file 1: Table S3 and Table S4).

Eight agronomic traits in the 9 SSSLs were investigated in two cropping seasons. Compared with HJX74, most of the traits in the SSSLs had no significant difference, except the plant height in SG25, the heading date in SG153, and so on (Additional file 1: Table S5).

\section{Two QTLs for SER Were Mapped on Chromosome 1}

Four SSSLs with high SER, SG18, SG22, SG23 and SG25, carried substituted segments on chromosome 1 . SG18 had a substituted segment at the interval from markers RM140 to RM5853 with the estimated length of 10,741.8 kb (Fig. 1c and Additional file 1: Table S4). Therefore, the substituted segment of SG18 carried a QTL for SER, named qSER-1a.

Three other SSSLs, SG22, SG23 and SG25, carried substituted segments in the region of 29,385.9-41,082.0 $\mathrm{kb}$ and the substituted segments overlapped each other. SG22 had the shortest substituted segment with the estimated length of $2611.3 \mathrm{~kb}$ from markers RM403 to RM6648. The estimated lengths of substituted segments 


\section{$\mathbf{a}$}

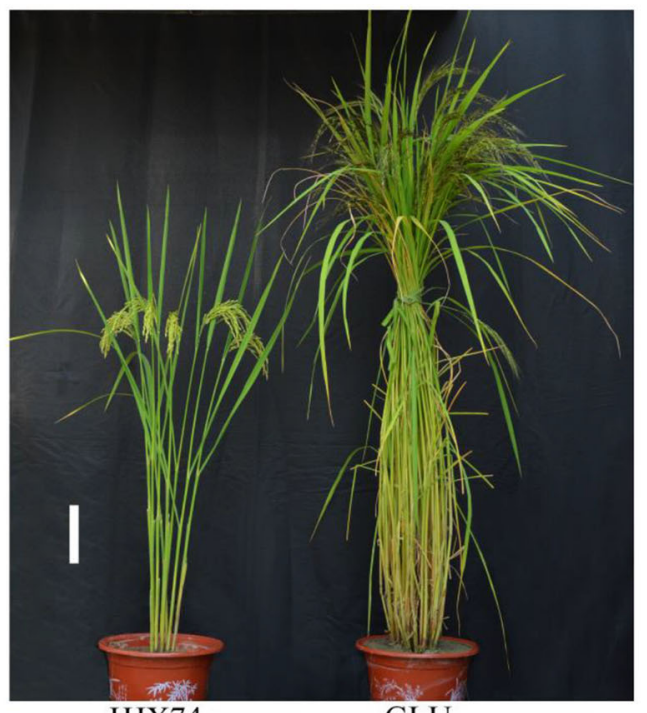

HJX74

GLU b

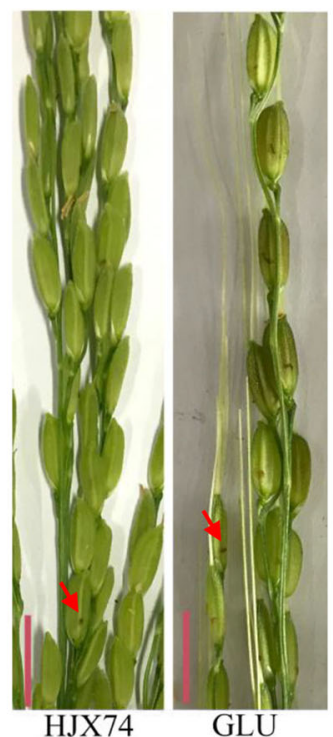

HJX74
GLU
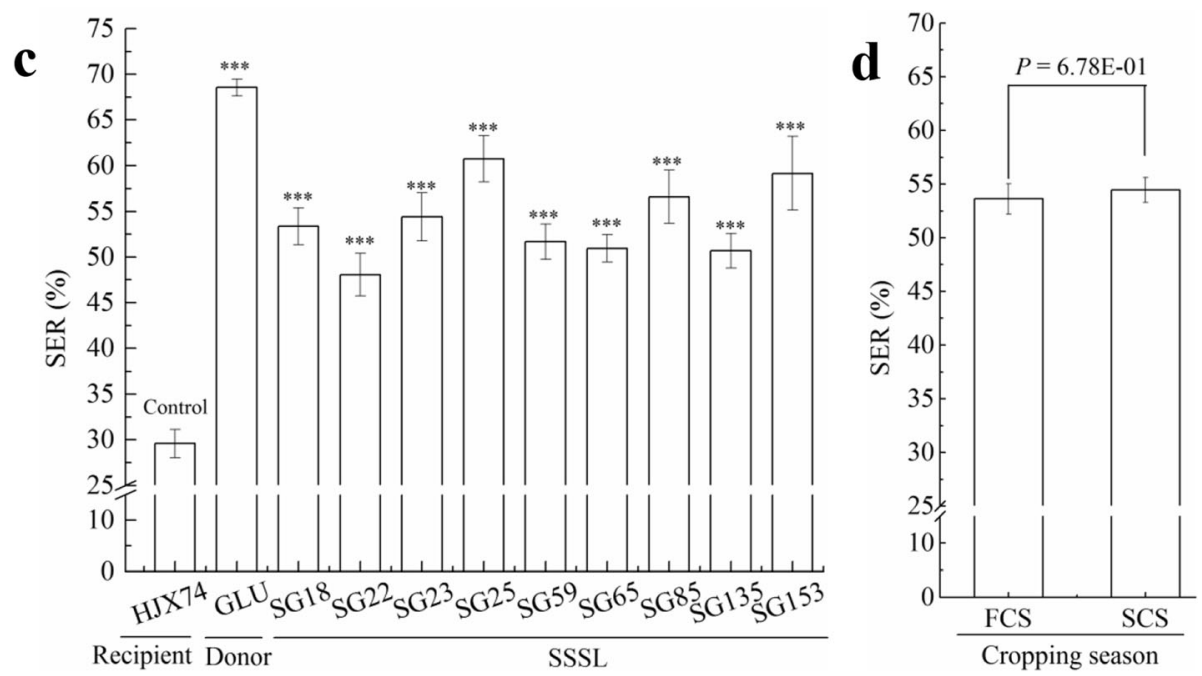

Fig. 1 Stigma exsertion of the SSSLs and their parents. a Plant type of the parents. Scale bar, $15 \mathrm{~cm}$. $\mathbf{b}$ Phenotype of exserted stigmas of the parents. The red arrow points to the exserted stigmas. Scale bar, $1 \mathrm{~cm}$. c SER in 9 SSSLs and their parents. Data are presented as mean \pm S.E. of five cropping seasons. One-way ANOVA, LSD, two-tailed, ${ }^{* * *} p$-value $\leq 0.001$. d Comparison of SER between FCS and SCS. Mean \pm S.E., two-tailed, two sample Student's t test. GLU: Oryza glumaepatula. SER: stigma exsertion rate. SSSL: single segment substitution line. FCS: first cropping season. SCS: second cropping season

were $5874.5 \mathrm{~kb}$ in SG23, and 11,059.7 kb in SG25. These results indicated that the three SSSLs carried another QTL for SER, named $q S E R-1 b$, which was located in the substituted segment of SG22 with an estimated interval of $2611.3 \mathrm{~kb}$ (Fig. 2 and Additional file 1: Table S4).

Because the $q S E R-1 b$ was linked to $S d 1$, SG25 carried the $S d 1$ in the substituted segment from O. glumaepatula and showed tall in plant height with $160.3 \mathrm{~cm}$ in FCS and $135.7 \mathrm{~cm}$ in SCS. SG22 and SG23 carried shorter substituted segments without $S d 1$ from $O$. glumaepatula. Although SG22 and SG23 were semi-dwarf in plant height, the SER had no significant difference with SG25 (Fig. 2 and Additional file 1: Table S5). These results indicated that the SER of $q S E R-1 b$ was not affected by plant height.

\section{Two QTLs for SER Were Mapped on Chromosome 3}

Two SSSLs with high SER, SG59 and SG65, carried substituted segments on chromosome 3. SG59 had a substituted segment at the interval from markers RM282 to InDel3 with the estimated length of $4632.1 \mathrm{~kb}$ (Fig. 1c and Additional file 1: Table S4). Therefore, the substituted segment of SG59 carried a QTL for SER, named $q S E R-3 a$. 


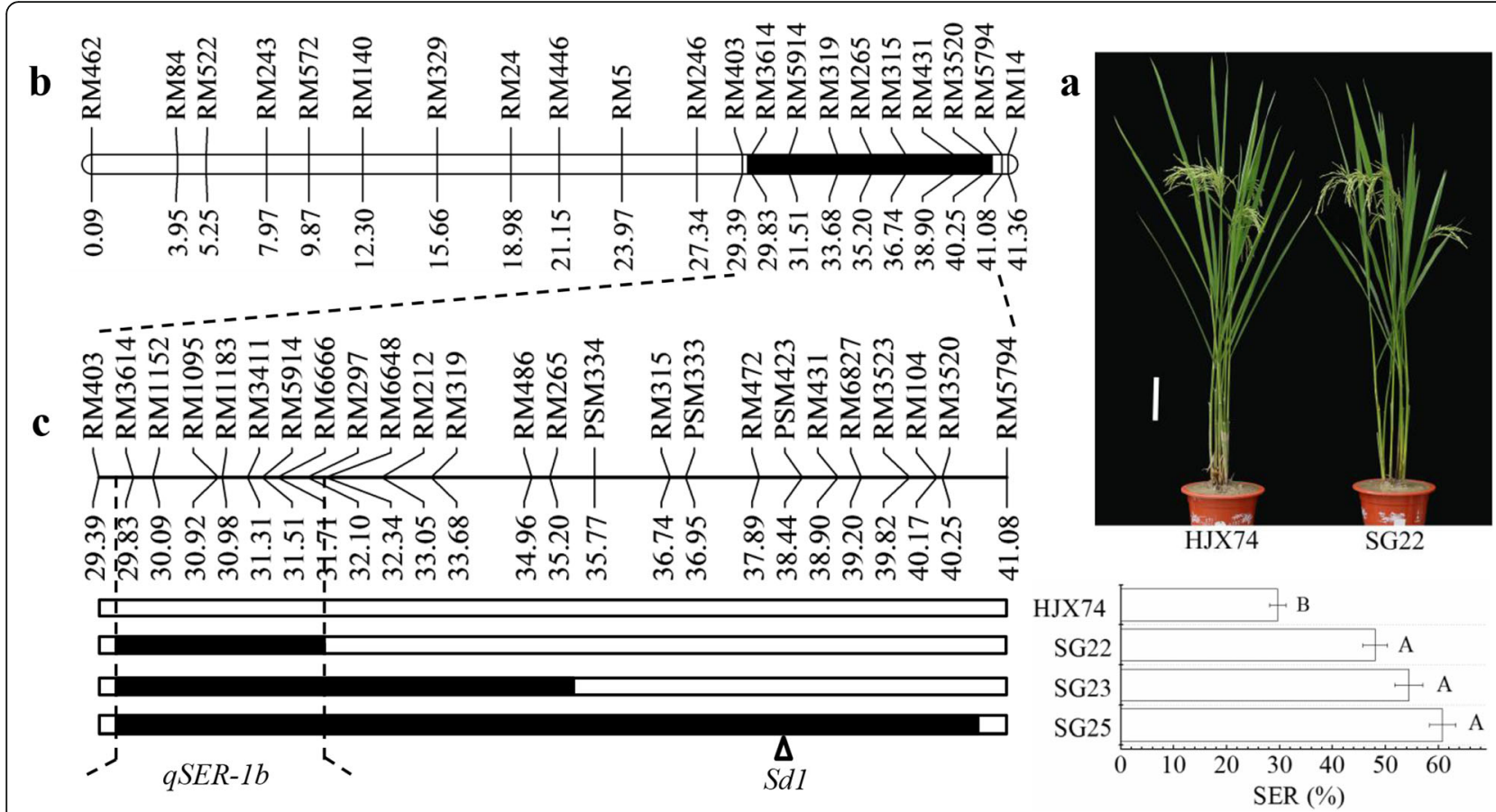

Fig. 2 Substitution mapping of qSER-16. a Plant type of HJX74 and SG22. Scale bar, $15 \mathrm{~cm}$. b The region of substituted segments of three SSSLS, SG22, SG23 and SG25, on chromosome 1. c Substitution mapping of aSER-1b, showing position of substituted segments and stigma exsertion rate (SER) of the SSSLS with HJX74 as the control. White and black blocks on chromosomes represent genotypes of HJX74 and donor, respectively. Threshold, $a=0.001$, one-way ANOVA, Duncan, two-tailed. The triangle represents the locus of Sd1 (Sasaki et al. 2002)

The SSSL SG65 carried a substituted segment at the interval of $26,747.5-35,843.1 \mathrm{~kb}$ on chromosome 3 . The substituted segment was in a different region from that in SG59. Therefore, SG65 had another QTL for SER, qSER$3 b$, in its substituted segment (Fig. 3a-b, Additional file 1: Table S4).

To narrow the interval of $q S E R-3 b, 5$ secondary SSSLs were developed from a $F_{2: 3}$ population derived from the cross of HJX74/SG65. The secondary SSSL, SG65-14, carried a substituted segment in the region from markers RM15678 to ID03MC07 on the left. Two of the secondary SSSLs, SG65-48 and SG65-61, had substituted segments in the regions from PSM132 and ID03MC22 to RM442 on the right, respectively. The three secondary SSSLs showed low SER as HJX74. Other two secondary SSSLs, SG65-9 and SG65-55, carrying substituted segments covering the region from markers ID03MC07 to ID03MC22 showed high SER as SG65. These results indicated that the $q S E R-3 b$ was narrowed to an estimated interval of $898.8 \mathrm{~kb}$ between markers ID03MC07 and ID03MC22 (Fig. 3c).

Using the RM168 marker at the $q S E R-3 b$ interval, Chisquare test was carried out in a $\mathrm{F}_{2}$ population of 80 individuals. The results revealed that the segregation ratio of three genotypes of the marker fit 1:2:1 $\left(\chi^{2}=0.60<\chi_{0.01,2}^{2}=9.21\right)$, and the heterozygous genotype showed incomplete dominance (Fig. 3d).

\section{Secondary Substitution Mapping of $q$ SER- 9}

One SSSL, SG135, carried a substituted segment on chromosome 9. Therefore, the substituted segment had a QTL for SER, qSER-9. To further map qSER-9, $5 \mathrm{sec}$ ondary SSSLs were developed from a $\mathrm{F}_{2: 3}$ population from the cross of HJX74/SG135. Two of the secondary SSSLs, SG135-26 and SG135-30, carried respectively substituted segments in the regions from ID09M06 to ID09M34 and RM105 on the left. The secondary SSSL, SG135-6, had a substituted segment in the region from ID09M23 to RM3600 on the right. The three secondary SSSLs showed low SER as HJX74. Two other secondary SSSLs, SG135-44 and SG135-68, carried substituted segments from ID09M34 to ID09M23, and had high SER as SG135. These results indicated that the qSER-9 was delimited to an interval between ID09M34 and ID09M23 with $551.9 \mathrm{~kb}$ estimated length (Fig. 4a-c).

Chi-square test of the RM6839 marker genotypes in a $\mathrm{F}_{2}$ population of 80 individuals revealed that the segregation ratio of the three genotypes was 1:2:1 ( $\chi^{2}=$ $0.48<\chi_{0.01,2}^{2}=9.21$ ) and the heterozygous genotype showed incomplete dominance (Fig. 4d).

\section{Other QTLs for SER Identified in the SSSLs}

One SSSL, SG85, carried a substituted segment from markers RM7444 to RM291 on chromosome 5 with the estimated length of $16,410.5 \mathrm{~kb}$. Therefore, the SSSL had 


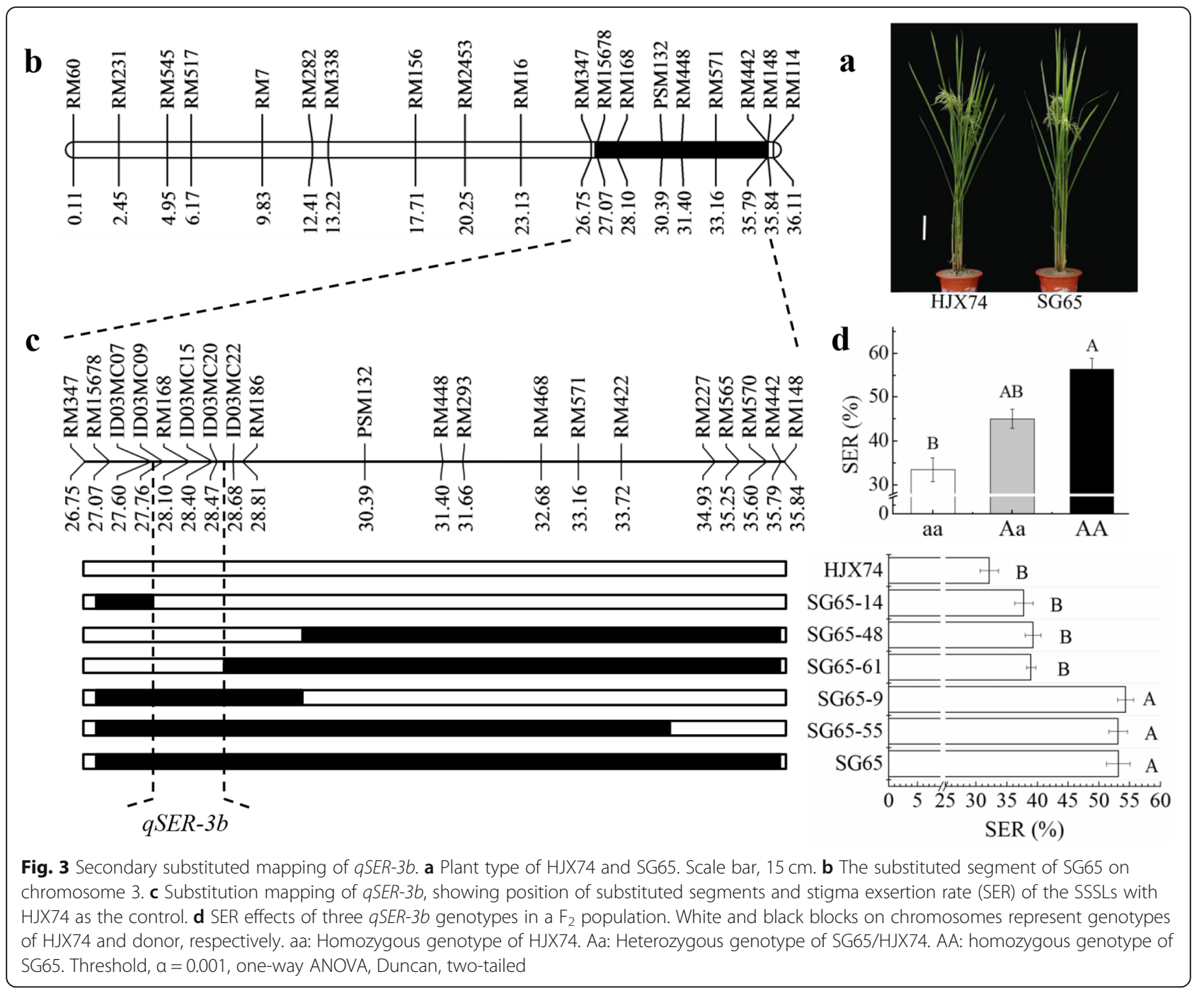

a QTL for SER, qSER-5, in the substituted segment (Fig. 1c and Additional file 1: Table S4).

Another SSSL, SG153, carried a substituted segment from markers RM484 to RM25886 on chromosome 10 with the estimated length of $1467.0 \mathrm{~kb}$ and had a QTL for SER, qSER-10, in the substituted segment (Fig. 1c and Additional file 1: Table S4).

Summarily, a total of 7 QTLs for SER was mapped on 5 chromosomes. Among of them, chromosomes 1 and 3 each carried two of the QTLs, and chromosomes 5, 9 and 10 each carried one of the QTLs (Fig. 5).

\section{The Additive Effects of QTLs for SER Identified in the SSSLS}

The additive effects of the 7 QTLs for SER, $q S E R-1 a$, qSER-1b, qSER-3a, qSER-3b, qSER-5, qSER-9 and qSER10 , ranged from $10.6 \%$ to $14.8 \%$. Among of them, $q S E R-$ 10 had the highest additive effect of $14.8 \%$ (Table 1).

\section{Discussion}

In the past two decades, many of QTLs controlling stigma exsertion were identified in rice genome. The QTLs were distributed across all 12 chromosomes (Marathi and Jena 2015; Zhou et al. 2017a; Liu et al. 2019). Among of them, a limited number of QTLs for SER were identified from the wild Oryza species, O. rufipogon (Xiong et al. 1999; Li et al. 2001; Uga et al. 2003a; Huang et al. 2012; Bakti and Tanaka 2019) and O. longistaminata ( $\mathrm{Li}$ et al. 2010). In the present study, we detected 7 QTLs of SER on 5 chromosomes using a set of SSSLs derived from O. glumaepatula (Fig. 5). On chromosome 1, qSER-1a and $q S E R-1 b$ are novel loci, in which region no other QTLs for SER were reported previously. On chromosome 3, qSER-3a was located in the QTL cluster of $q E S 3$ (Miyata et al. 2007), PES-3 (Yue et al. 2009), qSPE3 (Feng et al. 2010) and qSSE3 (Li et al. 2014a). $q S E R-3 b$ was mapped in the region locating of $q T S E-3 b$ (Li et al. 2014b) and qSER-3.2 (Xu et al. 2019). 

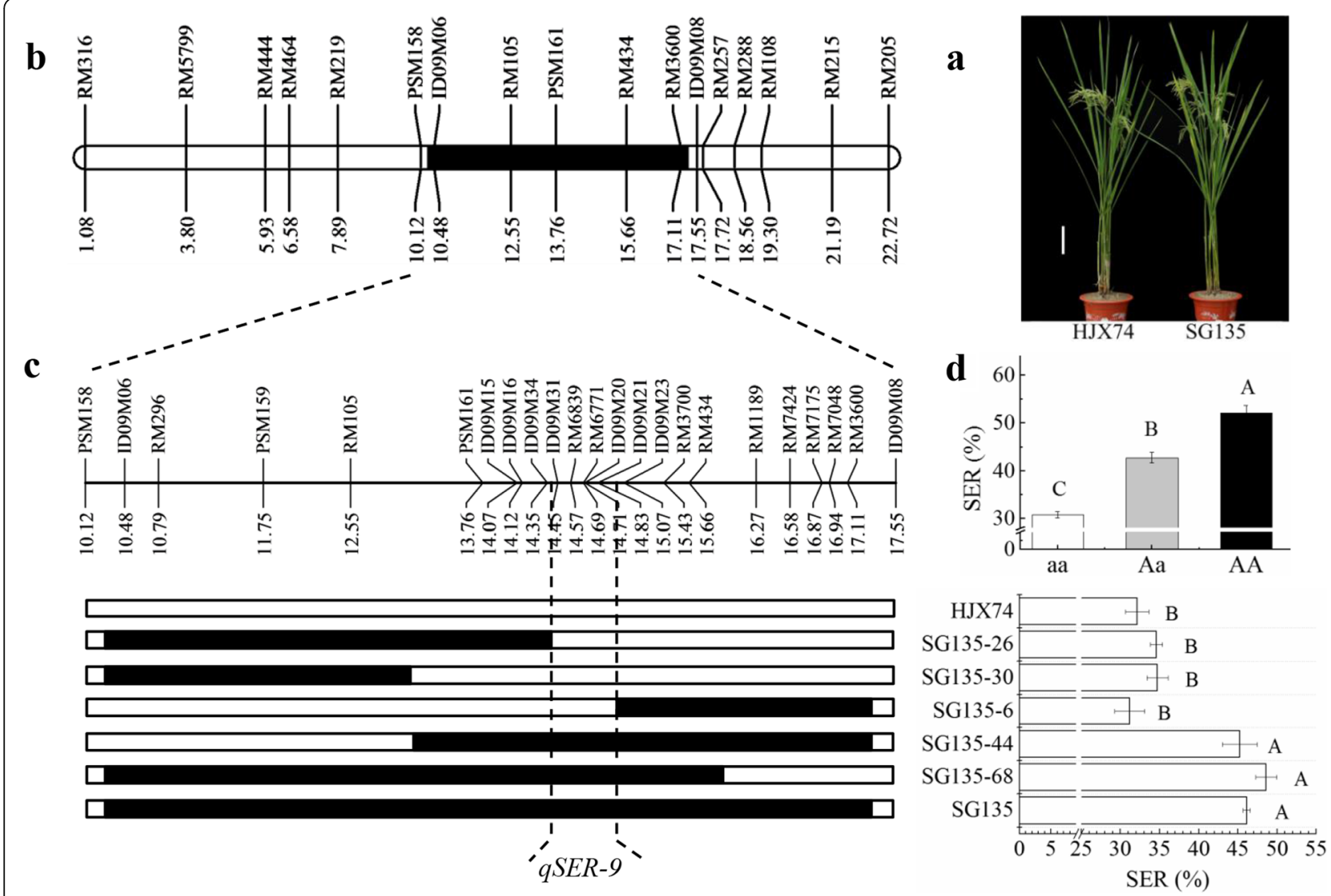

Fig. 4 Secondary substitution mapping of qSER-9. a, Plant type of HJX74 and SG135. Scale bar, $15 \mathrm{~cm}$. b. The substituted segment of SG135 on chromosome 9. c, Substitution mapping of aSER-9, showing position of substituted segments and stigma exsertion rate (SER) of the SSSLS with HJX74 as the control. d, SER effects of three qSER-9 genotypes in a $F_{2}$ population. White and black blocks on chromosomes represent genotypes of HJX74 and donor, respectively. aa: Homozygous genotype of HJX74. Aa: Heterozygous genotype of SG135/HJX74. AA: homozygous genotype of SG135. Threshold, $a=0.001$, one-way ANOVA, Duncan, two-tailed

On chromosome 5, the region of $q S E R-5$ covered those of qPEST-5 (Li et al. 2001), qPES-5 (Deng et al. 2010), qTSE-5a (Li et al. 2014b), TSE (Zhou et al. 2017a) and qSER-5.1 (Xu et al. 2019). On chromosome 9, the qSER9 region overlapped partly with the segment of $q P E S-9$ from T821B, an introgression line from O. longistaminata ( $\mathrm{Li}$ et al. 2010). In addition, the qSER-10 interval on chromosome 10 was overlapped with the region of qDSE-10 ( $\mathrm{Li}$ et al. 2014a). Although most of the loci identified in this study were located in the same regions as the previously reported loci, it is worth noting that the additive effects of the loci identified from O. glumaepatula were greater. The additive effects of the seven loci identified in this study were between $10.6 \%$ and $14.8 \%$ (Table 1), while those of the previously reported loci were usually less than $8.0 \%$. It indicated that the QTLs from O. glumaepatula had major effects on SER, which will be favorable for molecular breeding of MSLs with greater ability for outcrossing.

During the process of domestication, cultivated rice has already lost some traits of natural outcrossing
(Parmar et al. 1979). Wild Oryza species have a strong outcrossing ability due to their larger stigma, longer style, greater exsertion of the stigma, and longer periods of spikelet opening (Marathi et al. 2015; Marathi and Jena 2015). Previous studies revealed dominant differences for SER and floral traits between cultivated rice and wild rice (Virmani and Athwal 1973; Uga et al. 2003b). It was found that O. glumaepatula had longer of stigma length than cultivated rice (Marathi et al. 2015). In the present study, we identified 7 QTLs for SER with major effects from the SSSLs derived from O. glumaepatula (Table 1). It indicated that the favorable alleles for outcrossing traits, which had been lost in cultivated rice, could be mined in wild Oryza species.

It is well documented that the GS3 gene not only determines seed length but also exerts a pleiotropic effect on stigma length and exsertion, such that plants with the gs3 allele often have long kernels and higher SER (Miyata et al. 2007; Zhou et al. 2017a; Xu et al. 2019). It was found that the introduction of the GS3 gene into a japonica rice (with the gs3 allele) significantly reduced 


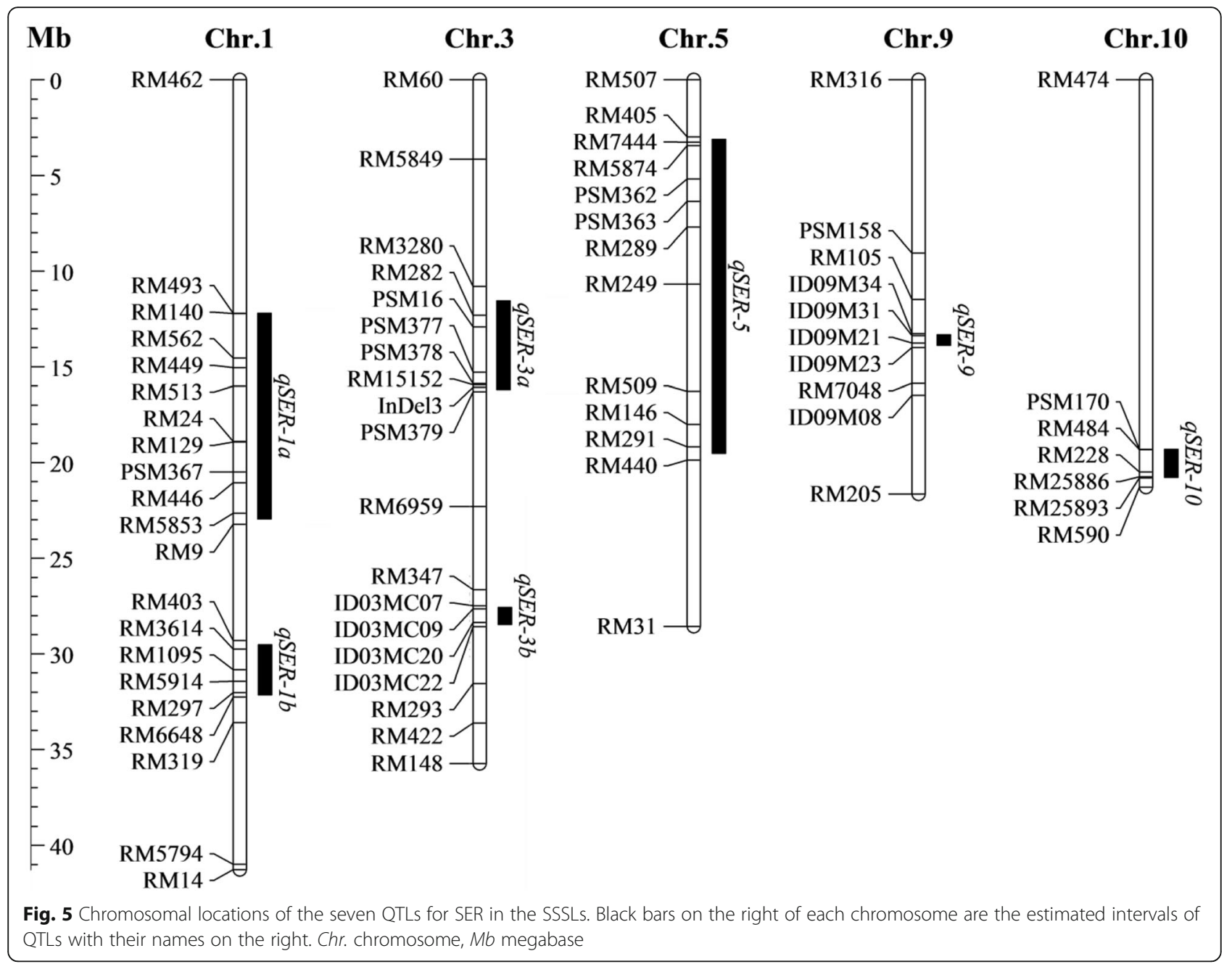

stigma exsertion in transgene-positive plants, suggesting that GS3 influences stigma exsertion (Takano-Kai et al. 2011). However, there are rice accessions with very low SER that also carry the gs 3 allele, suggesting that the beneficial effect of $g s 3$ on stigma exsertion is dependent on genetic background (Xu et al. 2019). Zhou et al. (2017a) found that rice accessions with the GW5gs3 combination had the highest stigma exsertion but still much lower than that of wild rice, indicating other loci contributions in rice domestication. In our research, HJX74 with GW5GS3 genotype showed low SER, while the SSSLs carrying QTLs of SER on its substituted segments showed high SER (Fig. 1c and Fig. 5). These results indicated that the high SER in the SSSLs was independent on the gs 3 gene. Furthermore, most of the SSSLs showed no significant difference in grain size with

Table 1 Additive effects of QTLs for stigma exsertion rate detected in the SSSLS

\begin{tabular}{|c|c|c|c|c|c|c|}
\hline QTL & Chr. & Interval (kb) & Estimated length (kb) & Maximum length (kb) & $P$ value & A (\%) \\
\hline aSER-1a & 1 & $12,291.6-23,033.4$ & $10,741.8$ & $11,044.9$ & $1.8 \mathrm{E}-09$ & $11.9 \pm 0.8$ \\
\hline$a S E R-1 b$ & 1 & $29,608.2-32,219.6$ & 2611.3 & 2952.5 & $1.2 \mathrm{E}-12$ & $12.4 \pm 1.8$ \\
\hline aSER-3a & 3 & $11,656.8-16,288.9$ & 4632.1 & 5502.7 & $1.2 \mathrm{E}-08$ & $11.1 \pm 1.1$ \\
\hline aSER-3b & 3 & $27,677.1-28,575.9$ & 898.8 & 1087.9 & 2.7E-08 & $10.7 \pm 1.2$ \\
\hline QSER-5 & 5 & $3216.5-19,626.9$ & $16,410.5$ & $16,901.8$ & $4.9 \mathrm{E}-11$ & $13.5 \pm 1.6$ \\
\hline QSER-9 & 9 & $14,398.5-14,950.4$ & 551.9 & 718.7 & 3.6E-08 & $10.6 \pm 1.0$ \\
\hline qSER-10 & 10 & $21,132.3-22,599.3$ & 1467.0 & 1504.7 & $2.9 \mathrm{E}-12$ & $14.8 \pm 2.0$ \\
\hline
\end{tabular}

$A$ additive effect, $A$ was represented as mean \pm S.E. in five cropping seasons. $P$ value indicates significant degree of SER between SSSL(s) and HJX74, determined by one-way ANOVA, LSD, two-tailed 
HJX74 (Additional file 1: Table S5). Therefore, the high SER in the SSSLs wasn't significantly affected by grain size.

In the past two decades, we have constructed a SSSL library by the using of HJX74 as the recipient and 43 accessions from 7 species, including 5 wild Oryza species of AA genome as donors (Zhang et al. 2004; Xi et al. 2006; Zhang 2019). The SSSLs have been used to map QTLs (Zhang et al. 2012; Yang et al. 2016; Zhou et al. 2017b), to clone genes (Wang et al. 2012; Sui et al. 2019), and to analyze allelic variation (Teng et al. 2012; Cai et al. 2013). Based on the SSSL library, we have developed a platform of breeding by design for cytoplasmic male sterility (CMS) lines and restorer lines, and a series of CMS lines and restorer lines were developed (Dai et al. 2015, 2016; Luan et al. 2019). The QTLs for SER detected in the present study will be used to develop CMS lines with strong outcrossing ability under the platform of SSSL library. Because SER was controlled by multiple QTLs and each QTL had only partial effect (Fig. 1c and Table 1), it is necessary to pyramid multiple QTLs to develop CMS lines with strong crossing ability. Although the effect of QTLs was affected by environments (Additional file 1: Table S2), the SER of plants had no significant difference between FCS and SCS (Fig. 1d). Therefore, CMS lines pyramiding the QTLs of SER will be applied to practical hybrid seed production both in FCS and in SCS. In addition, enhancing of outcrossing ability will be helpful to raise grain yield in hybrid rice. It is worth noting that the heterozygous genotype of the QTLs for SER showed incomplete dominance (Fig. 3d and Fig. 4d). Therefore, pyramiding of the QTLs for SER both in CMS lines and in restorer lines will help to raise the seed set of hybrid rice.

\section{Conclusion}

Using the SSSLs derived from O. glumaepatula, seven major QTLs controlling SER, qSER-1a, qSER-1b, qSER$3 a, q S E R-3 b, q S E R-5, q S E R-9$ and $q S E R-10$, were located on 5 chromosomes by substitution mapping. qSER-1a and $q S E R-1 b$ are novel loci for SER, in which regions no other QTLs of SER were reported previously. Compared with the previously reported loci, the 7 QTLs have major additive effects. The major QTLs will be favorable for molecular breeding of MSLs with strong outcrossing ability. The favorable alleles for outcrossing traits, which were lost in cultivated rice, could be mined in the wild Oryza species.

\section{Methods}

\section{Materials and Filed Experiment}

The set of SSSLs derived from O. glumaepatula (IRGC104387), an accession of the wild species indigenous to Brazil (Zhao et al. 2019) and their parents HJX74 and O. glumaepatula were grown in the paddy fields at the experimental farm of South China Agricultural University in Guangzhou $\left(23^{\circ} 07^{\prime} \mathrm{N}, 113^{\circ} 15^{\prime} \mathrm{E}\right)$, China. The materials were grown in 5 cropping seasons from 2016 to 2018, two cropping seasons per year, FCS and SCS. Single-seedling transplanting was applied in field experiment. Each plot had four rows with 10 single plants per row. Standard cultivation practices and controlling of diseases and insect pests were followed the typical methods in South China.

\section{Molecular Markers and PCR Protocol}

SSR markers labeled "RM" were selected from online resources (https://archive.gramene.org/markers/). The "PSM" and "Indel" markers were designed using the software of Primer Premier 5.0 (Lalitha 2000). DNA was extracted from the fresh leaves of each plant referencing a reported method (Murray and Thompson 1980). The PCR products were amplified and analyzed on 6\% denatured polyacrylamide gel, and detected using the silver staining using the methods described by Fang et al. (2019).

\section{Phenotyping of Traits and Statistical Analysis}

The stigma exsertion (SE) was subdivided into single stigma exsertion (SSE) and dual stigma exsertion (DSE). The SER refers to total stigma exsertion rate, including single stigma exsertion rate and dual stigma exsertion rate. For investigating SER, 8-10 main panicles were sampled from plants in 1-2 days after flourishing florescence of florets. The spikelet numbers of SSE and DSE per panicle were counted based on visual exserted stigmas (Xu et al. 2019). Grain traits were measured by using the YTS rice phenotypic facility (Yang et al. 2014). Differences of traits were determined using one-way ANOVA. SPSS 23.0 and OriginPro 9.0 (https://www.originlab.com) were applied to analyze the experimental data.

\section{Mapping of QTLs}

The lengths of substituted segments of SSSLs were calculated by the previous method (Xi et al. 2006; He et al. 2017; Zhao et al. 2019). The minimum length $\left(L_{\min }\right)$ of a substituted segment refers to the length between two markers of donor genotype at the end of the substitution segment. The maximum length $\left(\mathrm{L}_{\max }\right)$ refers to the length between two markers of background genotype flanking the substitution segment. The estimated length $\left(\mathrm{L}_{\text {est }}\right)=\left(\mathrm{L}_{\min }+\mathrm{L}_{\max }\right) / 2$. The QTLs for SER were mapped by the substitution mapping method (Eshed and Zamir 1995; Wissuwa et al. 2002). Linkage maps of markers were drawn by using MapChart2.2 (https://www.wur.nl/ en/show/Mapchart.htm). QTLs were named by the method of McCouch et al. (1997). 


\section{Supplementary information}

Supplementary information accompanies this paper at https://doi.org/10. 1186/s12284-020-00397-1.

Additional file 1: Table S1. Stigma exsertion rate of the 9 SSSLs in 5 cropping seasons. Table S2. Analysis of variance based on a fixed-effect model of the 9 SSSLs in 5 cropping seasons. Table S3. Developed markers used to detect the substituted segments of the SSSLS. Table S4. Substituted segments detected by markers in the SSSLs. Table S5. Phenotypes of agronomic traits in the SSSLS.

\section{Abbreviations}

CMS: Cytoplasmic male sterility; DSE: Dual stigma exsertion; FCS: First cropping season; MSL: Male sterility line; QTL: Quantitative trait locus; SCS: Second cropping season; SER: Stigma exsertion rate; SSE: Single stigma exsertion; SSSL: Single-segment substitution line

\section{Acknowledgments}

Not applicable.

\section{Authors' Contributions}

GZ and XF conceived and designed the experiments and supervised the research works. QT performed most of the experiments and prepared the data and the draft of manuscript. TZ, YN, MZ, XL, XL, WY and ZY performed a part of experiments. $H Z, R Z, G L, S W$ and $G Z$ participated in material development. GZ analyzed the data and wrote the manuscript. All authors read and approved the final manuscript.

\section{Funding}

This work was supported by grants from the National Natural Science Foundation of China (91435207 and 91735304), and from the Guangzhou Science and Technology Program Key Projects (201607020040 and 201804020086).

\section{Availability of Data and Materials}

All data generated or analyzed during this study are included in this published article and its additional information files.

\section{Ethics Approval and Consent to Participate}

Not applicable.

\section{Consent for Publication}

Not applicable.

\section{Competing Interests}

The authors declare that they have no competing interests.

Received: 7 April 2020 Accepted: 27 May 2020

Published online: 09 June 2020

\section{References}

Bakti C, Tanaka J (2019) Detection of dominant QTLs for stigma exsertion ratio in rice derived from Oryza rufipogon accession 'W0120'. Breed Sci 69:143-150

Bhatia D, Joshi S, Das A, Vikal Y, Sahi GK, Neelam K, Kaur K, Singh K (2017) Introgression of yield component traits in rice (Oryza sativa ssp. indica) through interspecific hybridization. Crop Sci 57:1557-1573

Cai J, Liao QP, Dai ZJ, Zhu HT, Zeng RZ, Zhang ZM, Zhang GQ (2013) Allelic differentiations and effects of the Rf3 and Rf4 genes on fertility restoration in rice with wild abortive cytoplasmic male sterility. Biol Plant 57:274-280

Dai Z, Lu Q, Luan X, Cai J, Zhu H, Liu Z, Zeng R, Zhang Z, Wang S, Zheng L, Li J, Zhang G (2015) Development of a platform for breeding by design of CMS lines based on an SSSL library in rice (Oryza sativa L.). Euphytica 205:63-72

Dai Z, Lu Q, Luan X, Ouyang L, Guo J, Liang J, Zhu H, Wang W, Wang S, Zeng R, Liu Z, Zhang Z, Zhu X, Zhang G (2016) Development of a platform for breeding by design of CMS restorer lines based on an SSSL library in rice (Oryza sativa L.). Breed Sci 66:768-775

Deng YD, Ying JZ, Shi YY, Xiao CL, Zhang HQ (2010) Mapping of QTLs for percentage of exserted stigma in rice. J Hunan Agric Univ 36:373-376 (In Chinese with English abstract)
Doi K, Yasui H, Yoshimura A (2008) Genetic variation in rice. Curr Opin Plant Biol 11:144-148

Eshed Y, Zamir D (1995) An introgression line population of Lycopersicon pennellii in the cultivated tomato enables the identification and fine mapping of yield-associated QTL. Genetics 141:1147-1162

Fang C, Li L, He R, Wang D, Wang M, Hu Q, Ma Q, Qin K, Feng X, Zhang G, Fu X, Liu Z (2019) Identification of S23 causing both interspecific hybrid male sterility and environment-conditioned male sterility in rice. Rice 12:10

Feng LL, Jing YH, Huang C, Xu ZJ, Chen WF (2010) QTL analysis of percentage of exserted stigma in rice (Oryza sativa L.). North Rice 40:20-22 (In Chinese with English abstract)

Fitzgerald MA, McCouch SR, Hall RD (2009) Not just a grain of rice: the quest for quality. Trends Plant Sci 14:133-139

He N, Wu R, Pan X, Peng L, Sun K, Zou T, Zhu H, Zeng R, Liu Z, Liu G, Wang S, Zhang G, Fu X (2017) Development and trait evaluation of chromosome single-segment substitution lines of $O$. meridionalis in the background of $O$. sativa. Euphytica 213:281

Huang X, Kurata N, Wei X, Wang Z, Wang A, Zhao Q, Zhao Y, Liu K, Lu H, Li W, Guo Y, Lu Y, Zhou C, Fan D, Weng Q, Zhu C, Huang T, Zhang L, Wang Y, Feng L, Furuumi H, Kubo T, Miyabayashi T, Yuan X, Xu Q, Dong G, Zhan Q, Li C, Fujiyama A, Toyoda A, Lu T, Feng Q, Qian Q, Li J, Han B (2012) A map of rice genome variation reveals the origin of cultivated rice. Nature 490:497501

Kato H, Namai H (1987) Floral characteristcs and environmental factors for increasing natural outcrossing rate for $F_{1}$ hybrid seed production of rice Oryza sativa L. Japan J Breed 37:318-330

Lalitha S (2000) Primer premier 5. Biotech Software Int Rep 1:270-272

Li C, Sun CQ, Mu P, Chen L, Wang XK (2001) QTL analysis of anther length and ratio of stigma exsertion, two key traits of classification for cultivated rice (Oryza sativa L.) and common wild rice (Oryza rufipogon Griff.). Acta Genet $\operatorname{Sin} 28: 746-751$

Li HB, Gao FY, Zeng LH, Li QX, Lu XJ, Li ZH, Ren JS, Su XW, Ren GJ (2010) QTL analysis of rice stigma morphology using an introgression line from Oryza longistaminata L. Mol Plant Breed 8:1082-1089 (In Chinese with English abstract)

Li P, Feng F, Zhang Q, Chao Y, Gao G, He Y (2014a) Genetic mapping and validation of quantitative trait loci for stigma exsertion rate in rice. Mol Breed 34:2131-2138

Li P, Su G, Feng F, Wang P, Yu S, He Y (2014b) Mapping of minor quantitative trait loci (QTLs) conferring fertility restoration of wild abortive cytoplasmic male sterility and QTLs conferring stigma exsertion in rice. Plant Breed 133: $722-727$

Liu Y, Zhang A, Wang F, Kong D, Li M, Bi J, Zhang F, Wang J, Luo X, Pan Z, Yu X, Liu G, Luo L (2019) Fine mapping a quantitative trait locus, qSER-7, that controls stigma exsertion rate in rice (Oryza sativa L). Rice 12:46

Luan X, Dai Z, Yang W, Tan Q, Lu Q, Guo J, Zhu H, Liu G, Wang S, Zhang G (2019) Breeding by design of CMS lines on the platform of SSSL library in rice. Mol Breed 39:126

Marathi B, Jena KK (2015) Floral traits to enhance outcrossing for higher hybrid seed production in rice: present status and future prospects. Euphytica 201: $1-14$

Marathi B, Ramos J, Hechanova SL, Oane RH, Jena KK (2015) SNP genotyping and characterization of pistil traits revealing a distinct phylogenetic relationship among the species of Oryza. Euphytica 201:131-148

McCouch SR, Cho YG, Yano M, Paul E, Blinstrub M, Morishima H, Kinosita T (1997) Report on QTL nomenclature. Rice Genet Newsl 14:11-13

Miyata M, Yamamoto T, Komori T, Nitta N (2007) Marker-assisted selection and evaluation of the QTL for stigma exsertion under japonica rice genetic background. Theor Appl Genet 114:539-548

Murray MG, Thompson WF (1980) Rapid isolation of high molecular weight plant DNA. Nucleic Acids Res 8:4321-4325

Oka HI, Morishima H (1967) Variations in the breeding systems of wild rice, Oryza perennis. Evolution 21:249-258

Parmar KS, Siddiq EA, Swaminathan MS (1979) Variation in components of flowering behavior of rice. Indian J Genet Plant Breed 39:542-550

Qian Q, Guo L, Smith SM, Li J (2016) Breeding high-yield superior quality hybrid super rice by rational design. Natl Sci Rev 3:283-294

Sakata M, Yamagata Y, Doi K, Yoshimura A (2014) Two linked genes on rice chromosome 2 for $F_{1}$ pollen sterility in a hybrid between Oryza sativa and 0 . glumaepatula. Breed Sci 64:309-320

Sano Y (1994) Pollen-killers in rice. Japan J Breed 44:298 
Santos KFDN, Silveira RDD, Martin-Didonet CCG, Brondani C (2013) Storage protein profile and amino acid content in wild rice Oryza glumaepatula. Pesq Agropec Bras 48:66-72

Sasaki A, Ashikari M, Ueguchi-Tanaka M, Itoh H, Nishimura A, Swapan D, Ishiyama K, Saito T, Kobayashi M, Khush GS, Kitano H, Matsuoka M (2002) Green revolution: a mutant gibberellin-synthesis gene in rice. Nature 416:701-702

Sasayama D, Okishio T, Hirano T, Fukayama H, Hatanaka T, Akimoto M, Azuma T (2018) Internodal elongation under submergence in the Amazonian wild rice species Oryza glumaepatula: the growth response is induced by hypoxia but not by ethylene. Plant Growth Regul 85:123-132

Sobrizal MY, Sanchez PL, Ikeda K, Yoshimura A (2000a) Identification of a gene for male gamete abortion in backcross progeny of Oryza sativa and Oryza glumaepatula. Rice Genet Newsl 17:59-61

Sobrizal MY, Sanchez PL, Ikeda K, Yoshimura A (2000b) Mapping of $F_{1}$ pollen semi-sterility gene found in backcross progeny of Oryza sativa L. and Oryza glumaepatula Steud. Rice Genet Newsl 17:61-63

Sobrizal MY, Yoshimura A (2001) Mapping of a gene for pollen semi-sterility on chromosome 8 of rice. Rice Genet Newsl 18:59-61

Sobrizal MY, Yoshimura A (2002) Mapping of pollen semi-sterility gene, S28(t), on rice chromosome 4. Rice Genet Newsl 19:80-82

Stein JC, Yu Y, Copetti D, Zwickl DJ, Zhang L, Zhang C, Chougule K, Gao D, Iwata A, Goicoechea JL, Wei S, Wang J, Liao Y, Wang M, Jacquemin J, Becker C, Kudrna D, Zhang J, Londono CEM, Song X, Lee S, Sanchez P, Zuccolo A, Ammiraju JSS, Talag J, Danowitz A, Rivera LF, Gschwend AR, Noutsos C, Wu C, Kao S, Zeng J, Wei F, Zhao Q, Feng Q, El Baidouri M, Carpentier M, Lasserre E, Cooke R, Rosa Farias DD, Da Maia LC, Dos Santos RS, Nyberg KG, McNally KL, Mauleon R, Alexandrov N, Schmutz J, Flowers D, Fan C, Weigel D, Jena KK, Wicker T, Chen M, Han B, Henry R, Hsing YC, Kurata N, de Oliveira AC, Panaud O, Jackson SA, Machado CA, Sanderson MJ, Long M, Ware D, Wing RA (2018) Genomes of 13 domesticated and wild rice relatives highlight genetic conservation, turnover and innovation across the genus Oryza. Nat Genet 50:285-296

Sui F, Zhao D, Zhu H, Gong Y, Tang Z, Huang XY, Zhang G, Zhao FJ (2019) Mapbased cloning of a new total loss-of-function allele of OsHMA3 causing high cadmium accumulation in rice grain. J Exp Bot 70:2857-2871

Takano-Kai N, Doi K, Yoshimura A (2011) GS3 participates in stigma exsertion as well as seed length in rice. Breed Sci 61:244-250

Teng B, Zeng R, Wang Y, Liu Z, Zhang Z, Zhu H, Ding X, Li W, Zhang G (2012) Detection of allelic variation at the $W x$ locus with single-segment substitution lines in rice (Oryza sativa L.). Mol Breed 30:583-595

Uga Y, Fukuta Y, Cai HW, Iwata H, Ohsawa R, Morishima H, Fujimura T (2003a) Mapping QTLs influencing rice floral morphology using recombinant inbred lines derived from a cross between Oryza sativa L. and Oryza rufipogon Griff. Theor Appl Genet 107:218-226

Uga Y, Fukuta Y, Ohsawa R, Fujimura T (2003b) Variations of floral traits in Asian cultivated rice (Oryza sativa L.) and its wild relatives (O. rufipogon Griff.). Breed Sci 53:345-352

Virmani SS, Athwal DS (1973) Genetic variability in floral characteristics influencing outcrossing in Oryza sativa L. Crop Sci 13:66-67

Wang S, Wu K, Yuan Q, Liu X, Liu Z, Lin X, Zeng R, Zhu H, Dong G, Qian Q, Zhang G, Fu X (2012) Control of grain size, shape and quality by OsSPL16 in rice. Nat Genet 44:950-954

Wissuwa M, Wegner J, Ae N, Yano M (2002) Substitution mapping of Pup 1: a major QTL increasing phosphorus uptake of rice from a phosphorus-deficient soil. Theor Appl Genet 105:890-897

Xi Z, He F, Zeng R, Zhang Z, Ding X, Li W, Zhang G (2006) Development of a wide population of chromosome single-segment substitution lines in the genetic background of an elite cultivar of rice (Oryza sativa L.). Genome 49: 476-484

Xiong LZ, Liu KD, Dai XK, Xu CG, Zhang Q (1999) Identification of genetic factors controlling domestication-related traits of rice using an $\mathrm{F}_{2}$ population of a cross between Oryza sativa and O. rufipogon. Theor Appl Genet 98:243-251

Xu S, Zheng Y, Liu Y, Guo X, Tan Y, Qian Q, Shu Q, Huang J (2019) Identification of a major quantitative trait locus and its candidate underlying genetic variation for rice stigma exsertion rate. Crop J 7:350-359

Xu YB, Shen ZT (1988) Receptivity of exserted stigmas. Int Rice Res Newsl 13:7-8

Yang T, Zhang S, Zhao J, Liu Q, Huang Z, Mao X, Dong J, Wang X, Zhang G, Liu B (2016) Identification and pyramiding of QTLs for cold tolerance at the bud bursting and the seedling stages by use of single segment substitution lines in rice (Oryza sativa L.). Mol Breed 36:96
Yang W, Guo Z, Huang C, Duan L, Chen G, Jiang N, Fang W, Feng H, Xie W, Lian X, Wang G, Luo Q, Zhang Q, Liu Q, Xiong L (2014) Combining highthroughput phenotyping and genome-wide association studies to reveal natural genetic variation in rice. Nat Commun 5:5087

Yue GH, Mei HW, Pan BR, Lou J, Li MS, Luo LJ (2009) Mapping of QTLs affecting stigma exsertion rate of Huhan $1 \mathrm{~B}$ as a CMS maintainer of upland hybrid rice. Acta Agriculturae Zhejiangensis 21:241-245 (In Chinese with English abstract)

Zhang GQ (2019) The platform of breeding by design based on the SSSL library in rice. Hereditas (Beijing) 41:754-760 (In Chinese with English abstract)

Zhang GQ, Zeng RZ, Zhang ZM, Ding XH, Li WT, Liu GF, He FH, Tulukdar A, Huang CF, Xi ZY, Qin LJ, Shi JQ, Zhao FM, Feng MJ, Shan ZL, Chen L, Guo $X Q$, Zhu HT, Lu YG (2004) The construction of a library of single segment substitution lines in rice (Oryza sativa L.). Rice Genet Newsl 21:85-87

Zhang Y, Yang J, Shan Z, Shen C, Qiao W, Zhu X, Xie Q, Zhu H, Zhang Z, Zeng R (2012) Substitution mapping of QTLS for blast resistance with SSSLs in rice (Oryza sativa L.). Euphytica 184:141-150

Zhang Y, Zhou J, Yang Y, Li J, Xu P, Deng X, Deng W, Wu Z, Tao D (2015) A novel gene responsible for erect panicle from Oryza glumaepatula. Euphytica 205:739-745

Zhao H, Sun L, Xiong T, Wang Z, Liao Y, Zou T, Zheng M, Zhang Z, Pan X, He N, Zhang G, Zhu H, Liu Z, He P, Fu X (2019) Genetic characterization of the chromosome single-segment substitution lines of $O$. glumaepatula and $O$. barthii and identification of QTLs for yield-related traits. Mol Breed 39:51

Zhou H, Li P, Xie W, Hussain S, Li Y, Xia D, Zhao H, Sun S, Chen J, Ye H, Hou J, Zhao D, Gao G, Zhang Q, Wang G, Lian X, Xiao J, Yu S, Li X, He Y (2017a) Genome-wide association analyses reveal the genetic basis of stigma exsertion in rice. Mol Plant 10:634-644

Zhou Y, Xie Y, Cai J, Liu C, Zhu H, Jiang R, Zhong Y, Zhang G, Tan B, Liu G, Fu X, Liu Z, Wang S, Zhang G, Zeng R (2017b) Substitution mapping of QTLS controlling seed dormancy using single segment substitution lines derived from multiple cultivated rice donors in seven cropping seasons. Theor Appl Genet 130:1191-1205

\section{Publisher's Note}

Springer Nature remains neutral with regard to jurisdictional claims in published maps and institutional affiliations.

\section{Submit your manuscript to a SpringerOpen ${ }^{\circ}$ journal and benefit from:}

- Convenient online submission

- Rigorous peer review

- Open access: articles freely available online

High visibility within the field

- Retaining the copyright to your article

Submit your next manuscript at $>$ springeropen.com 